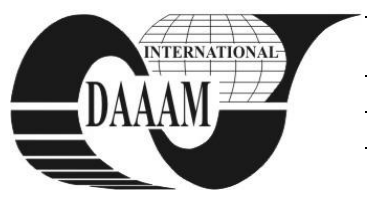

\title{
INFLUENCE OF HORIZON LENGTH OF MODEL PREDICTIVE CONTROL WITH QUADRATIC CRITERIUM
}

\author{
VESELY, I[vo] \& ZAMECNIK, D[usan]
}

\begin{abstract}
This work deals with various settings of prediction and control horizon of objective function in model predictive control. Both horizons have a significant impact on quality and on the effort of optimization. Longer-term prediction allows us to better capture the dynamic of the system, but on the other hand, with increasing predictive horizon, the complexity of optimization grows exponentially. In this paper, we present the results and conclusions from our simulations of first-order constrained system, which has been optimized in the sense of the regulator simplicity to quality control ratio. The system was put to the test by reference tracking on the unit step. According to our results, the optimal values of both prediction and control horizons is 2 - increasing either of these values does not bring any improvement in the quadratic criterion sense.
\end{abstract}

Key words: model model predictive control, prediction horizon, control horizon, quadratic criterion

\section{INTRODUCTION}

Model predictive control (MPC) covers a group of algorithms intended for finite horizon optimization of multipleinput and multiple-output (MIMO) systems with time-delay with constrains. The greatest advantage of MPC-based algorithms is the ability to deal with the given constraints and automatized controller design. The main drawback is the high computational demand. The increasing number of MPC applications in industry is shown in paper (Qin \& Badgwell, 2003) and the use of MPC for induction machine control in theory (Geyer, 2009) and implementation (Papafotiou, 2009).

Main idea of prediction control is to utilize system output behavior based on model of plant. Hence a MPC control law has the following components by (Rossiter, 2004):

1) It depends on predicted behavior.

2) Prediction is computed using a process model.

3) The current control action is a result of optimization.

4) The most commonly feature in predictive control is Receding horizon control (RHC) strategy which introduces a feedback in a straightforward predictive control. It means that control action is updated at every sampling instant.

Explicit optimization offline controller is explained e.g. (Bemporad et al., 2001). Main idea is to remove a huge computational effort into offline part. In online part, the explicit controller only searches out state space and chooses an appropriate solution which enables faster solution in evaluation. Explicit controller calculates for all combinations of states all control laws (during design phase of controller). It solves not $\mathrm{QP}$, but multi-parametric QP (mp-QP), where initial state vector is a parameter. The state space is divided into linear separated regions (given by length of prediction horizon and number of state variables and constraints).

The solution of optimization (explicit controller) is the resulting control law. The control law (control action) is a piecewise affine and continuous function of actual state vector in form $\mathbf{u}^{*}=\mathbf{K}_{\mathrm{i}} \mathbf{x}+q_{\mathrm{i}}$ for each $i$-th region.

The complexity of MPC controller depends on length of prediction and control horizon exponentially.

\section{PREDICTION MODEL}

A vital component of every MPC controller is a discretetime model (with constraints) and a cost function (mostly quadratic). The cost function is defined over a finite horizon (predicted horizon) with length $N$ steps.

Linear discrete-time first order model in common state space form was used to predict future behavior.

$$
\begin{aligned}
\mathbf{x}(t+1) & =\mathbf{A} \mathbf{x}(t)+\mathbf{B u}(t) \\
\mathbf{y}(t) & =\mathbf{C x}(t)+\mathbf{D u}(t)
\end{aligned}
$$

by subject the box constraints:

$$
y_{\min } \leq y \leq y_{\max }, u_{\min } \leq u \leq u_{\max }
$$

It is defining which control action is optimal. A total system response is defined by sum of both forced and free response depends only on the past control actions and initial state, respectively. Control action has form as follow.

$$
\mathbf{u}(t, N)=\left[\mathbf{u}(t)^{T}, \mathbf{u}^{T}(t+1), \ldots, \mathbf{u}^{T}(t+N-1)\right]^{T}
$$

The state response has a normal and a matrix form.

$$
\begin{aligned}
& \mathbf{x}(t+k)=\mathbf{A}^{k} \mathbf{x}(t)+\sum_{i=t}^{t+k-1} \mathbf{A}^{i} \mathbf{B} \mathbf{u}(k-1-i) \\
& \mathbf{x}(t+k)=\mathbf{P} \mathbf{x}(t)+\mathbf{H u}(t)
\end{aligned}
$$

, where $N$ is a finite prediction horizon. A quadratic cost function that covers this prediction horizon is defined by (5) and in matrix form by (6).

\section{COST FUNCTION}

$$
\begin{aligned}
J(\mathbf{x}(t), \mathbf{u}(t, N))= & \frac{1}{2} \sum_{k=t}^{t+N-}\left[\mathbf{x}^{T}(k) \mathbf{Q} \mathbf{x}(k)+\mathbf{u}^{T}(k) \mathbf{R u}(k)\right]+ \\
& +\frac{1}{2} \mathbf{x}^{T}(k+N) \mathbf{Q} \mathbf{x}(k+N) \\
J(\mathbf{x}(t), \mathbf{u}(t, N))= & \frac{1}{2} \mathbf{u}^{T}(t, N) \mathbf{T} \mathbf{u}(t, N)+\mathbf{x}(t)^{T} \mathbf{F} \mathbf{u}(t, N)+ \\
& +\frac{1}{2} \mathbf{x}(t)^{T} \mathbf{Y} \mathbf{x}(t)
\end{aligned}
$$

where $\quad \mathbf{T}=\operatorname{diag}(\mathbf{R}, \ldots, \mathbf{R})+\mathbf{H}^{\mathrm{T}} \mathbf{O T}, \quad \mathbf{F}=\mathbf{P}^{\mathrm{T}} \mathbf{O H}, \quad \mathbf{Y}=\mathbf{P}^{\mathrm{T}} \mathbf{O P}$, $\mathbf{O}=\operatorname{diag}\left(\mathbf{Q}, \ldots \mathbf{Q}, \mathbf{Q}_{\mathrm{N}}\right)$. Matrix $\mathbf{Q}$ penalizes output state over whole prediction horizon except last step. The terminal state is penalized by $\mathbf{Q}_{\mathrm{N}}$, where $\mathbf{Q}_{\mathrm{N}}>\mathbf{Q}$, to achieve better stability performance. Matrix R penalizes control action signal $\boldsymbol{u}$.

Optimal cntrol action for constrained system with quadratic cost function is defined by equation (7).

$$
\begin{aligned}
& \mathbf{u}_{t, N}^{*}(\mathbf{x}(t))=\arg \underbrace{\min }_{\mathbf{u}_{t, N}^{\min }}\left[\frac{1}{2} \mathbf{u}_{t, N}^{T} \mathbf{T} \mathbf{u}_{t, N}+\right. \\
& \left.+\mathbf{x}^{T} \mathbf{F} \mathbf{u}_{t, N} \mid \mathbf{G} \mathbf{u}_{t, N} \leq \mathbf{w}+\mathbf{E x}(t)\right]
\end{aligned}
$$

Control horizon $N_{C}\left(N_{C} \leq N\right)$ was introduced to decreases 
the degrees of freedom consequent less amount of computational effort is needed for an optimization. Without introducing control horizon is generated sequence of optimal control action $\boldsymbol{u}$ with length $N_{C}=N$ steps. If $N_{C}<N$ than only first $N_{C}$ steps is optimized and the others are keeping at the constant value (at the last optimized value). For detailed insight follow (Kvasnica, 2009).

\section{SIMULATION}

This concrete example was used in MPC for induction machine control (Zámečník et al., 2011).

Constraints are $-4<u<4,-1<y<1$. The matricies are: $\mathbf{A}=0.95$, $\mathbf{B}=0.05, \mathbf{C}=1$ and $\mathbf{D}=0$. This system is derived from first order system with time constant $\mathrm{T}=7,8 \mathrm{~ms}$, and gain equals one, and discretized by fast sample period $\mathrm{T}_{\mathrm{S}}=400 \mu \mathrm{s}$. The future response of model is determined exactly by control actions $\boldsymbol{u}$, model parameters and actual system state (by neglecting disturbances and model inaccuracy). Hard constraints cannot be exceeded, because the crash of numerical optimization (controller being in infeasibility region) occurs in the case of violation this constraints.

Simulink models are depicted at Fig. 1 and 2.

\subsection{MPC controller design}

Controller was designed with use of Multi-Parametric Toolbox (MPT) (Kvasnica et al., 2004) with tracking reference and following settings:

The norm of cost function was set to 2 , it implies to quadratic programming problem. Penalization matrices were set: $\mathbf{Q}=700, \mathbf{Q}_{\mathbf{N}}=1000$, and $\mathbf{R}=0.1$.

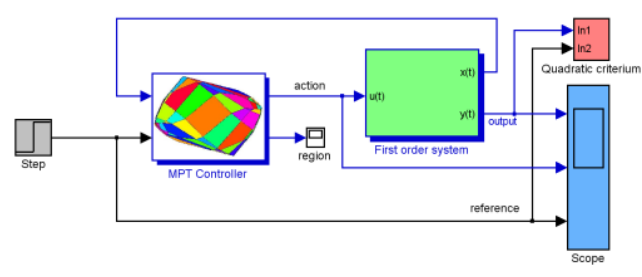

Fig. 1. Simulink scheme of whole experiment

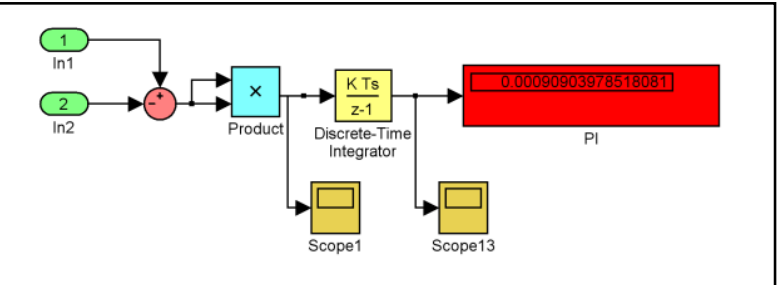

Fig. 2. Simulink scheme of quadratic criteria

\section{RESULTS OF SIMULATIONS}

The results of simulations for various lengths of prediction and control horizons are shown in Tab.1 and 2, where N/A means no feasibility control law found. Nominal value of quadratic criteria is $n=0.00116807$ and the others values are expressing relatively deffirence the actual value of criteria in comparison with this nominal value in promile $(1 / 1000)$. For example the value $-0.07 \mathrm{n}$ represents that value of quadratic criteria was approximately 0.07 promile better than nominal.

\begin{tabular}{|l|l|l|l|l|l|}
\hline \multirow{2}{*}{$\begin{array}{l}\text { Control } \\
\text { horizon }\end{array}$} & \multicolumn{4}{l}{ Prediction horizon } & \multicolumn{4}{l|}{} \\
\cline { 2 - 6 } & 1 & 2 & 3 & 4 & 5 \\
\hline 1 & N/A & N/A & N/A & N/A & N/A \\
\hline 2 & & $-0.62 \mathrm{n}$ & $1.3 \mathrm{n}$ & $4.6 \mathrm{n}$ & $7.6 \mathrm{n}$ \\
\hline 3 & & & $0.08 \mathrm{n}$ & $-0.07 \mathrm{n}$ & $-0.16 \mathrm{n}$ \\
\hline 4 & & & & $\mathrm{n}$ & $\mathrm{n}$ \\
\hline 5 & & & & & $\mathrm{n}$ \\
\hline
\end{tabular}

Tab. 1. Title of results for $N=1 \ldots 5$

\begin{tabular}{|c|c|c|c|c|c|}
\hline \multirow{2}{*}{$\begin{array}{l}\text { Control } \\
\text { horizon }\end{array}$} & \multicolumn{5}{|c|}{ Prediction horizon } \\
\hline & 6 & 7 & 8 & 9 & 10 \\
\hline 1 & $\mathrm{~N} / \mathrm{A}$ & N/A & $\mathrm{N} / \mathrm{A}$ & $\mathrm{N} / \mathrm{A}$ & $\mathrm{N} / \mathrm{A}$ \\
\hline 2 & $27.5 n$ & $54.2 n$ & $82 n$ & $131 n$ & $175 n$ \\
\hline 3 & $-0.21 n$ & $-0.31 n$ & $-0.36 n$ & $-0.40 n$ & $-0.44 n$ \\
\hline 4 & $n$ & $n$ & $n$ & $\mathrm{n}$ & $n$ \\
\hline 5 & $n$ & n & $n$ & $n$ & n \\
\hline 6 & $n$ & n & $\mathrm{n}$ & n & $n$ \\
\hline 7 & & $n$ & n & n & $n$ \\
\hline 8 & & & n & n & n \\
\hline 9 & & & & n & $\mathrm{n}$ \\
\hline 10 & & & & & $\mathrm{n}$ \\
\hline
\end{tabular}

Tab. 2. Title of results for $N=6 \ldots 10$

\section{CONCLUSION}

The results are showing that the control horizon $N_{C}>3$ is not better option, because the quadratic criteria of quality, in this case, is keeping at same nominal value.

The control horizon $N_{C}=2$ performanced the relatively poor, and with the increasing prediction horizon quality was decreasing. By contrast the control horizon $N_{C}=3$ performanced well and the performance was increasing with increasing prediction horizon

The best performance was reach at $N_{C}=2, N=2$, but it is not enoght serious setting for practice implementation, prediction is too short and it cannot cover reliable dynamics of system.

Detailed investigation with various systems with conditions nearer to practice, will be carrying out in further work.

\section{ACKNOWLEDGEMENTS}

This work was supported by grant "Research of Modern Methods and Approaches in Automation" from the Internal Grant Agency of Brno University of Technology (grant No. FEKT-S-11-6) .

\section{REFERENCES}

Bemporad, A; Morari, M.; Dua, V.; Efstratois, N. \& Pistikopoulos, N. (2001): The explicit linear quadratic regulator for constrained systems, Automatica, Vol. 38

Tøndel, P., Johansen, T. A., \& Bemporad, A. (2001a). An algorithm for multi-parametric quadratic programming and explicit MPC solutions. In Proceedings of the 40th IEEE Conference on Decision and Control. Orlando, FL, pp. 1199-1204

Qin, S.J. \& Badgwell,T.A (2003): A survey of model predictive control technology, In Control engeenering practice (11), pp. 733-746

Rossiter J.A (2004): Model-Based predictive control A practical approach, CRC Press, ISBN 0-8493-1291-4

Kvasnica, M.; Grieder, P. \& Baoti, M.(2004) Multi-Parametric Toolbox (MPT). Place: Automatic Control Laboratory, ETH Zurich, Switzerland. Available from: http://control.ee.ethz.ch/ mpt/ Accessed: 2011-05-13

Kvasnica, M. (2009) Real-Time Model Predictive Control via Multi-Parametric Programming : Theory and Tools. Saarbrucken : VDM Verlag,. 272p. ISBN 978-3639206449

Papafotiou, G.; Kley, J.; Papadopoulos, K.G.; Bohren, P.; Morari, M. (2009): Model Predictive Direct Torque Control-Part II: Implementation and Experimental Evaluation, Industrial Electronics, IEEE Transactions on , vol.56, no.6, pp.1906-1915

Geyer, T.; Papafotiou, G.; Morari, M. (2009): Model Predictive Direct Torque Control-Part I: Concept, Algorithm, and Analysis, Industrial Electronics, IEEE Transactions on, vol.56, no.6, pp.1894-1905

Zámečník, D.; Veselý, I. (2011) Model predictive current controller of induction machine, ElectroScope http://www.electroscope.zcu.cz, n.2, pp. 1-5. ISSN: 18024564 